\title{
Cyclic Analysis of Phonocardiogram Signals
}

\author{
A.Choklati, K. Sabri and M. Lahlimi \\ STIC laboratory, Faculty of sciences, University Chouaib Doukkali, El Jadida, Morocco \\ Emails: choklati.a@ucd.ac.ma, sabri.k@ucd.ac.ma, lahlimi.mounir@gmail.com
}

Received: 14 April 2017; Accepted: 01 August 2017; Published: 08 October 2017

\begin{abstract}
Acoustic vibrations of the heart in time domain correspond to phonocardiogram (PCG) signal. A PCG signal, in the healthy case, consists of two fundamental sounds $s_{1}$ and $s_{2}$ produced by the mechanical functioning of the heart. Abnormalities in the heart valves correspond to other cardiac sounds than $s_{1}$ and $s_{2}$. This makes PCG signal a valuable tool related to the track of heart diseases. Actually, the characterization and the analysis of PCG signals is being a fertile area of study and investigation. However, most of the topics which treated this area of research focused only on timefrequency analysis, without exploiting the periodic character of PCG signal due to the limitations of the PCG modeling. In this work, we propose a coherent mathematical model for PCG signals based on cyclostationarity and Gabor kernel. The motivation behind is to define a framework, utilizing cyclic statistic due to noise robustness, for a full description of PCG signals, which leads to an easy and efficient early identification of certain heart abnormalities. The validation of the proposed model and its capacity to reflect the heart functioning is tested over synthetic and real data sets.
\end{abstract}

Index Terms - Heart sound, phonocardiogram modeling, cyclostationarity, cyclic statistics, Gabor kernel, heart diseases.

\section{INTRODUCTION}

The Phonocardiogram signal (PCG) is a temporal representation of the acoustic sound of the heart vibration. It is a considerable source of information that can lead by its analysis, to the detection and the identification of several heart abnormalities. A PCG signal in the healthy case consists of two fundamental sounds, i.e. the first heart sound $s_{1}$ and the second heart sound $s_{2}$. These two fundamental sounds are derived from the mechanical functioning of the heart and are due to the closing of the valves and the turbulent passage of blood [14]. There are other sounds than $s_{1}$ and $s_{2}$ that may correspond to diseases or problems in the heart valves. Although some diseases tend to be recognized with difficulty, by using a stethoscope, they are all reflected on the patient PCG signal. That is why we resort to this type of signals to identify the state of the heart.
Regarding the analysis of digital PCG signals, all related works in this area are based on time-frequency analysis [12, 9]. To the best of our knowledge, the existing models do not exploit the periodic character of the heart functioning. As a matter of fact, the heart sound is a series of repeated mechanical actions. The repetition is not exactly periodic but is considered quasi-periodic. It means that the vibration wave recordings are cyclostationary $[1,13,16]$. Many natural or man-made processes in various fields arise from periodic phenomena, as mechanics, telecommunications, radio astronomy, econometric, ... These processes, although not periodic functions of time, give rise to random data whose statistics vary periodically with time and are called cyclostationary processes [20].

A stochastic signal $x(t)$ of mean $\mathrm{E}\{x(t)\}$ and timevarying autocorrelation function

$$
R_{x}(t, \tau)=\mathrm{E}\left\{x(t-\tau / 2) x^{*}(t+\tau / 2)\right\},
$$

where the superscript $*$ denotes complex conjugation, is said to be wide-sense cyclostationary with $\mathrm{T}_{0}$-period if both $\mathrm{E}\{x(t)\}$ and $R_{x}(t, \tau)$ are periodic over time $\mathrm{t}$ with $\mathrm{T}_{0}$-period [3], i.e. $\mathrm{E}\{x(t)\}=\mathrm{E}\left\{x\left(t+T_{0}\right)\right\}$ for all $\mathrm{t}$ $R_{x}(t, \tau)=R_{x}\left(t+T_{0}, \tau\right)$ for all $\mathrm{t}, \tau$.

The time-varying autocorrelation function is, thus, periodic over tand can be expanded in Fourier series:

$$
R_{x}(t, \tau)=\sum_{n=-\infty}^{+\infty} R_{x}^{n / T_{0}}(\tau) e^{j 2 \pi \frac{n}{T_{0}} t}
$$

where $R_{x}^{n / T_{0}}(\tau)$ is known as the cyclic autocorrelation function and is given by:

$$
R_{x}^{n / T_{0}}(\tau)=\frac{1}{T_{0}} \int_{-T_{0} / 2}^{T_{0} / 2} R_{x}(t, \tau) e^{-j 2 \pi \frac{n}{T_{0}} t} \mathrm{~d} t
$$

where $n / T_{0}, n \in \square$ are the cyclic frequencies.

The Fourier transform of the cyclic autocorrelation function with respect to the cyclic frequency $\alpha$ gives rise to the spectral correlation density function: 


$$
S_{x}^{\alpha}(f)=\int_{-\infty}^{+\infty} R_{x}^{\alpha}(\tau) e^{-j 2 \pi \alpha \tau} \mathrm{d} \tau
$$

In this context, several models representing the functioning of the heart sounds were presented in the literature [14, 18, 8]. However, these models are limited to a single cardiac cycle, and hence remain uncompleted. Thus, the purpose of this study is to introduce a coherent cyclostationary and Gabor kernel based model for a whole description of PCG signals. The proposed model takes into account the fluctuation of the heart beats from one cycle to another. The motivation behind is to define a framework, utilizing cyclic statistic due to noise robustness, for a full description of PCG signals, which leads to an easy and efficient early identification of certain heart abnormalities. The validation of the proposed model and its capacity to reflect the heart functioning is tested over synthetic and real data sets.

This paper is organized as follows. Section 2, describes the modeling of PCG signals. Section 3 concerns the analytical study of the proposed model, some simulation results are also presented in this section in order to confirm the theoretical analysis. Section 4 focuses on the validation of the computed cyclic statistics on synthetic and experimental PCG signals. Finally, Section 5 is dedicated to conclusions.

\section{PCG MODELING}

\section{A. Context}

A PCG beat mainly consists of two distinct sounds, $s_{1}$ and $s_{2}$, sample waveforms of which are depicted in Fig. 1 . The need of an adequate framework, for the detection and the classification of abnormalities in the heart functioning, has pushed researchers to be interested in the modeling of the PCG signals. Therefore, modeling the shape of the heart sounds has been studied by many authors: the chirp model [10, 11, 17], the damped sinusoidal model [5, 2], and the modified Prony model [6]. To our knowledge, all related models are limited to a single cardiac cycle and give no information about the behavior of heart sounds for the remaining cycles. The motivation of this work is to build up a mathematical model able to produce the shape and the quasi-periodic character of heart sounds.

\section{B. Established PCG Model}

Among the existing PCG models, the ones based on Gabor and Laplace kernels [18, 19, 7, 8, 10] seem to be more realistic. The Gabor kernel, which is actually a Gaussian-damped sinusoidal wave, offers the possibility, through five adjustable parameters $\left(a_{i}, \sigma_{i}, \mu_{i}, f_{i}\right.$ and $\left.\varphi_{i}\right)$, to exactly reproduce the shape of any heart beat.

The model of (1) makes use of two Gabor kernels to represent each heart sound $s_{1}$ and $s_{2}$. Therefore, one heart beat of PCG signal can be modeled with four kernels as given bellow (Fig. 1):

$$
\sum_{i \in\left[s_{1}^{ \pm} ; s_{2}^{ \pm}\right]} a_{i} \exp \left(-\frac{\left(t-\mu_{i}\right)^{2}}{2 \sigma_{i}^{2}}\right) \cos \left(2 \pi f_{i} t-\varphi_{i}\right)
$$

Where

- $f_{i}$ and $\varphi_{i}$ are respectively the frequency and the phase shift of the sinusoid terms.

- $a_{i}, \mu_{i}$, and $\sigma_{i}$ are successively the amplitude, the center and the width which represent the parameters of the Gaussian terms.

- $\quad \pm$ is a superscript indicating the two Gabor kernels which are used for modeling each heart sound, with $\left[s_{1}^{ \pm} ; s_{2}^{ \pm}\right]=\left[s_{1}^{-}, s_{1}^{+} ; s_{2}^{-}, s_{2}^{+}\right]$.

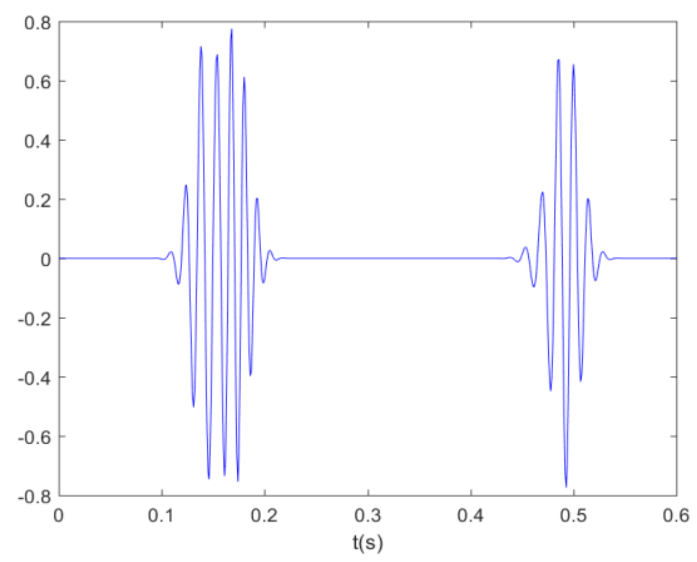

Fig.1. Example of healthy PCG signal for a single heart cycle of (1).

\section{Modified PCG Model}

The model of (1) represents the PCG signal for a single cardiac cycle. Unfortunately, this representation is not enough to make a full characterization of the heart in a limited time. Hence, the idea of the proposed model, to achieve a whole description is to jointly combine Gabor kernels, for modeling the shape of heart sounds, with some randomness to reproduce the fluctuations occurring in the heart functioning for every cardiac cycle. This combination leads to the model given by the following expression:

$$
z(t)=\sum_{i \in\left[s_{1}^{ \pm}, s_{2}^{ \pm}\right], n} a_{i, n} \exp \left(-\frac{\left(t-\mu_{i}-n T\right)^{2}}{2 \sigma_{i}^{2}}\right) \cos \left(2 \pi f_{i}(t-n T)-\varphi_{i, n}\right)
$$

Where

- The index $\mathrm{n}$ stands for the cardiac cycle.

- $T$ is the cardiac cycle duration.

The random behavior in $z(t)$ comes simultaneously from the parameters $a_{i, n}$ and $\varphi_{i, n}$. This means that the amplitude and the phase for each heart sound might 
change for any cardiac cycle. Where $a_{i, n}$ is the amplitude of Gabor kernel, for the ith heart sound and the nth cardiac cycle, which follows a Gaussian law $\mathrm{N}\left(\mu_{a i}, \sigma_{a i}^{2}\right)$ whereas the phase $\varphi_{i, n}$ follows a uniform law inside the interval $\left[\varphi_{i, 0}-\Delta \varphi, \varphi_{i, 0}+\Delta \varphi\right]$ with is $\Delta \varphi \in[0, \pi]$ and $\varphi_{i, 0}$ the $i t h$ initial phase. An example of the proposed model of (2) is given by Fig. 2 where the parameters are showed in Tab. 1.

Moreover $T=1 s, K=100$ cardiac cycles and the sampling frequency $f_{s}$ is set to. $1000 \mathrm{~Hz}$.

Table. 1. Mean values of the phonocardiogram (PCG) model parameters according to (2).

\begin{tabular}{|c|c|c|c|c|c|c|c|c|}
\hline & $\begin{array}{c}\mu_{a i} \\
(m v)\end{array}$ & $\begin{array}{c}\sigma_{a i} \\
(m v)\end{array}$ & $\begin{array}{l}\mu_{i} \\
(s)\end{array}$ & $\begin{array}{l}\sigma_{i} \\
(s)\end{array}$ & $\begin{array}{c}\varphi_{i, 0} \\
(\mathrm{rad})\end{array}$ & $\begin{array}{l}\Delta \varphi_{i} \\
(\mathrm{rad})\end{array}$ & $\begin{array}{c}f_{i} \\
(H z)\end{array}$ \\
\hline \multirow{4}{*}{ 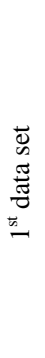 } & $s_{1}^{+}$ & 0.8 & 0.02 & 0.0414 & 0.0127 & 2.77 & $\frac{\pi}{10}$ & 66.66 \\
\hline & $s_{1}^{-}$ & 0.8 & 0.15 & 0.0716 & 0.0127 & 1.73 & $\frac{\pi}{10}$ & 78.85 \\
\hline & $s_{2}^{+}$ & 0.8 & 0.10 & 0.3836 & 0.0143 & 3.14 & $\frac{\pi}{10}$ & 66.92 \\
\hline & $s_{2}^{-}$ & 0.9 & 0.07 & 0.3883 & 0.0143 & 3.14 & $\frac{\pi}{10}$ & 71.19 \\
\hline
\end{tabular}

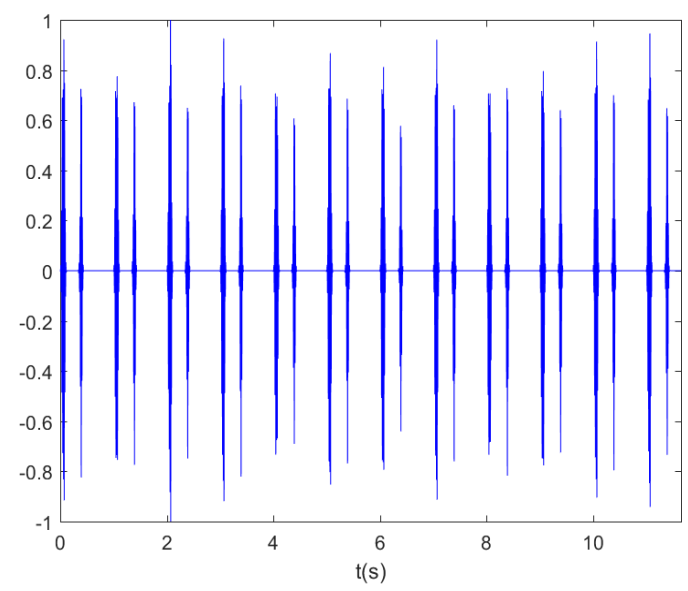

Fig.2. Example of a healthy PCG signal of (2), which shows the almost periodic behavior of PCG signals.

\section{CyClostationary ANALysis of THE PROPOSED PCG MODEL}

\section{A. $1^{\text {st }}$-Order and $2^{\text {nd }}$-Order Moments}

Let us check the wide-sense cyclostationarity for the proposed model using the previous definitions. We first start compute the $1^{\text {st }}$-order moment of $z(t)$ and, then, the time-varying autocorrelation function. The expectation $\mathrm{E}\{z(t)\}$ is given by:

$$
m_{z}(t)=\frac{\sin \Delta \varphi}{\Delta \varphi} \sum_{i \in\left[s_{1}^{ \pm}, s_{2}^{ \pm}\right], n} \mu_{a, i} \exp \left(-\frac{\left(t-\mu_{i}-n T\right)^{2}}{2 \sigma_{i}^{2}}\right) \cos \left(2 \pi f_{i}(t-n T)-\varphi_{i, 0}\right)
$$

$m_{z}(t)$ is T-periodic which indicates that $\mathrm{z}(t)$ is $1^{\text {st }}$ order cyclostationary. It should be noted that $m_{z}(t)$ converges to 0 when $\Delta \varphi$ moves toward $\pi$ as. $\Delta \varphi \in[0, \pi]$.

Besides, the computation of the time-varying autocorrelation function, of the PCG signal after removing the first order cyclostationarity, is given by the following relationship:

$$
\begin{aligned}
& R_{z}(t, \tau)=\sum_{i, n} \frac{\sigma_{a i}^{2}}{2}\left\{\cos \left(2 \pi f_{i} \tau\right)+\frac{\sin (2 \Delta \varphi)}{2 \Delta \varphi} \cos \left(4 \pi f_{i}(t-n T)-2 \varphi_{i, 0}\right)\right\} \\
& \exp \left(-\frac{\left(t-\mu_{i}-n T\right)^{2}}{\sigma_{i}^{2}}\right) \exp \left(-\frac{\tau^{2}}{4 \sigma_{i}^{2}}\right)
\end{aligned}
$$

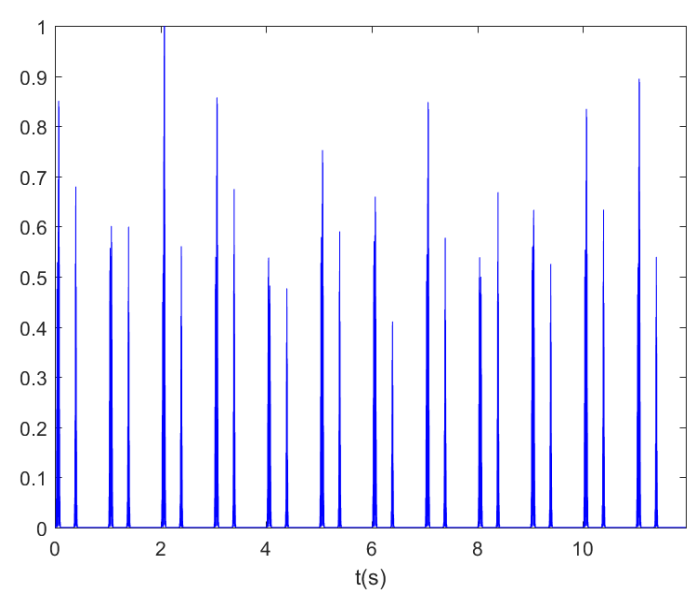

Fig.3. A numerical estimate of the time-varying autocorrelation function $R_{z}(t, \tau)$ for $\tau=0 s$, of the synthetic signal of Fig. 2. 
$R_{z}(t, \tau)$ is T-periodic as well as $\mathrm{E}\{z(t)\}$. Hence, we come to the conclusion that the signal of the proposed model of (2) is well wide-sense cyclostationary. Moreover, Fig. 3 reports a numerical estimator of $R_{z}(t, \tau)$, of the synthetic signal of Fig. 2 which confirms the previous theoretical result.

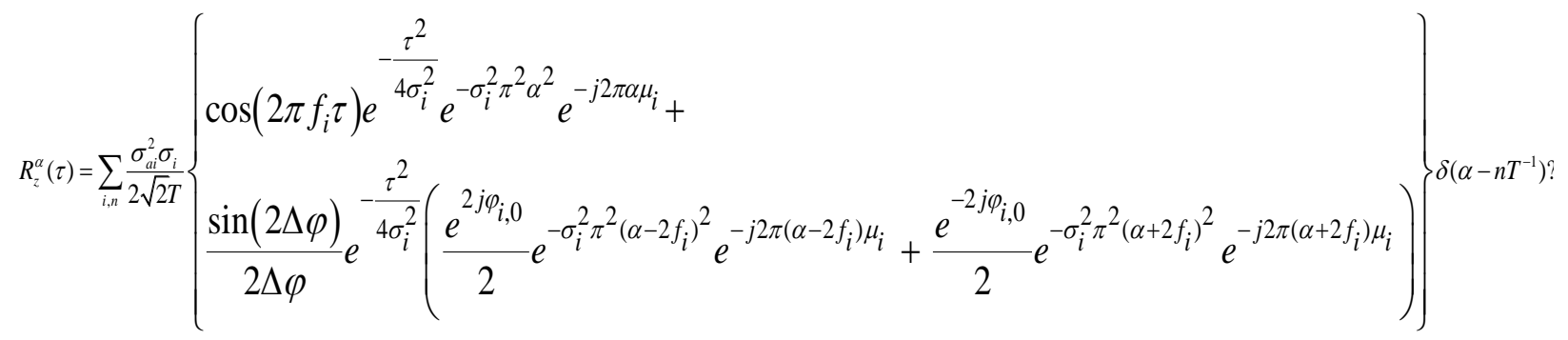

\section{B. Cyclic Autocorrelation Function $R_{z}^{\alpha}(\tau)$}

According to Gardner [3], the cyclic autocorrelation function can be defined by performing the Fourier transform of $R_{z}(t, \tau)$ with respect to t. Thus, the Fourier transform of $R_{z}(t, \tau)$ of (3) leads to:

\section{)}

With $\delta($.$) denotes the Dirac's delta.$

The first thing to note is that $R_{z}^{\alpha}(\tau)$ is $\alpha$-discrete and is nonzero only for the harmonics of $T^{-1}$. This point confirms the second order cyclostationarity of the model of (2). Also, the term $\frac{\sigma_{a i}^{2} \sigma_{i}}{2 \sqrt{2} T}$ increases when $\sigma_{a i}$ increases too, this will result in an increase of cyclostationarity. However, $\frac{\sin (2 \Delta \varphi)}{2 \Delta \varphi}$ decreases when $\Delta \varphi$ goes to $\pi$ which leads to a decrease of cyclostationarity.

Fig. 4 reports a numerical estimator of $R_{z}^{\alpha}(\tau)$ of the synthetic signal of Fig. 2. As expected from theory, the figure shows that the cyclic autocorrelation is nonzero only for the harmonics of $T^{-1}$ i.e $1 \mathrm{~Hz}$ which validates the previous theoretical results.

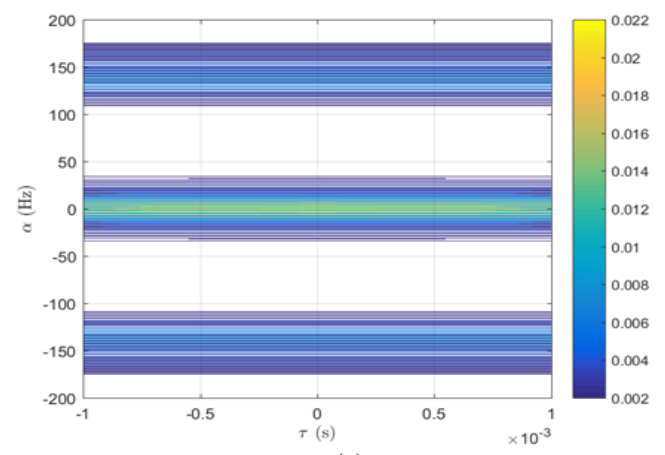

(a) 


\section{Cyclic Spectral Autocorrelation Function $S_{z}^{\alpha}(f)$}

$S_{z}^{\alpha}(f)$ represents another important second order cyclic statistic allowing the characterization in the $(f, \alpha)$-plan. As defined by Gardner [4], the cyclic spectral correlation of a cyclostationary random process in the broad sense is the Fourier transform of its cyclic correlation function with respect to $\tau$. So, the Fourier transform $R_{z}^{\alpha}(\tau)$ of (4) leads to:

$$
S_{z}^{\alpha}(f)=\sum_{i, n} \frac{\sigma_{a i}^{2} \sigma_{i}^{2}}{4 T}\left\{\begin{array}{l}
\left(e^{-4 \sigma_{i}^{2} \pi^{2}(f-f i)^{2}}+e^{-4 \sigma_{i}^{2} \pi^{2}(f+f i)^{2}}\right) e^{-\sigma_{i}^{2} \pi^{2} \alpha^{2}} e^{-j 2 \pi \alpha \mu_{i}}+ \\
\frac{\sin (2 \Delta \varphi)}{2 \Delta \varphi} e^{-4 \sigma_{i}^{2} \pi^{2} f^{2}}\left(e^{2 j \varphi_{i, 0}} e^{-\sigma_{i}^{2} \pi^{2}\left(\alpha-2 f_{i}\right)^{2}} e^{-j 2 \pi\left(\alpha-2 f_{i}\right) \mu_{i}}+e^{-2 j \varphi_{i, 0}} e^{-\sigma_{i}^{2} \pi^{2}\left(\alpha+2 f_{i}\right)^{2}} e^{\left.-j 2 \pi\left(\alpha+2 f_{i}\right) \mu_{i}\right)}\right.
\end{array}\right\} \delta\left(\alpha-n T^{-1}\right)
$$

It should be noted that for the zero cyclic frequency i.e. $\alpha=0$, the later relationship is reduced to the power spectrum density:

$$
S_{z}^{0}(f)=\sum_{i, n} \frac{\sigma_{a i}^{2} \sigma_{i}^{2}}{4 T}\left\{e^{-4 \sigma_{i}^{2} \pi^{2}(f-f i)^{2}}+e^{-4 \sigma_{i}^{2} \pi^{2}(f+f i)^{2}}+\frac{\sin (2 \Delta \varphi)}{2 \Delta \varphi} e^{-4 \sigma_{i}^{2} \pi^{2}\left(f^{2}+f_{i}^{2}\right)}\left(e^{2 j \varphi_{i, 0}} e^{j 4 \pi f_{i} \mu_{i}}+e^{-2 j \varphi_{i, 0}} e^{-j 4 \pi f_{i} \mu_{i}}\right)\right\}
$$

As it is shown by the relationship (5), the cyclic spectral correlation $S_{z}^{\alpha}(f)$ is $\alpha$-discrete and is nonzero only for $\alpha=n T^{-1}$ with resonances around $\pm 2 f_{i}$. Furthermore, $S_{z}^{\alpha}(f)$ is f-continuous and presents peaks in frequencies $\pm f_{i}$, with $i \in\left[s_{1}^{ \pm}, s_{2}^{ \pm}\right]$, where $f_{i}$ represents a characteristic frequency.

Moreover, Fig. 5 reports a numerical estimator of $S_{z}^{\alpha}(f)$ of the synthetic signal of Fig. 2 which confirms the effectiveness of the theoretical results mentioned previously.

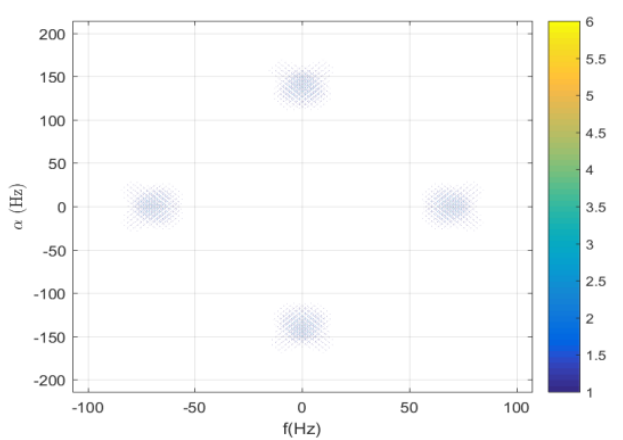

(a)

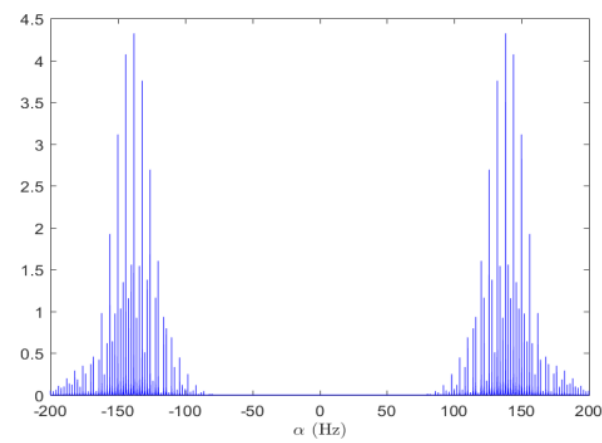

(b)

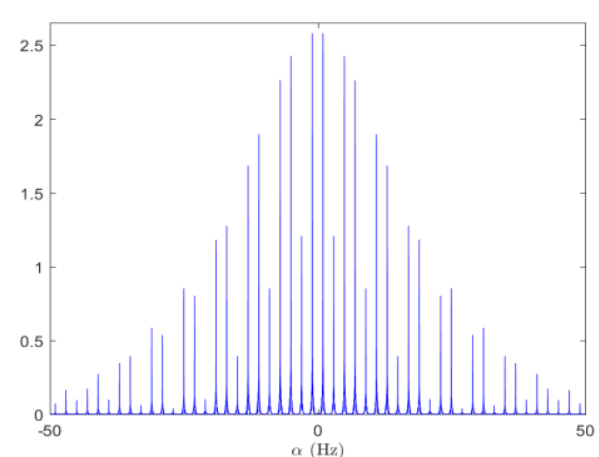

(c)

Fig.5. A numerical estimate of the spectral correlation density $S_{z}^{\alpha}(f)$ : (a) $S_{z}^{\alpha}(f)$ a function of $(\alpha, f),\left(\right.$ b) $S_{z}^{\alpha}(f)$ in the $\alpha$-plan for $f=0 H z$, (c) zoom of $S_{z}^{\alpha}(f)$ in axis $\alpha$ for $f=73.24 \mathrm{~Hz}$, of the synthetic signal of Fig. 2.

\section{EVALUATION ON SYNTHETIC AND EXPERIMENTAL PCG SignALS}

\section{A. Realistic Synthetic PCG Signals}

Additional simulations have been made in order to confirm the cyclostationary behavior of the mathematical model of (2) regarding its parameters. Actually, the parameters

$$
\mu_{a i}, \sigma_{a i}, \mu_{i}, \sigma_{i}, \varphi_{i, 0}, \Delta \varphi_{i} \text { and } f_{i}
$$

mentioned in subsection II.C, might vary from beat to beat and for each person i.e. these parameters represent the functioning of a unique heart. 
Two healthy hearts cannot have exactly the same parameters. Other data sets are necessary to understand the influence of these parameters on the PCG model and to evaluate how much cyclic statistics represent a coherent signature and characteristic even for different hearts (different parameters), and thereby a suitable to recognize healthy hearts. The parameters for the simulations are listed in Tab. 2. Moreover, an additive Gaussian noise is added such that the SNR is set to the desired values. The sampling frequency for the three signals is set to $1000 \mathrm{~Hz}$.

Table. 2. Parameters to generate three sets of realistic PCG signals according to (2).

\begin{tabular}{|c|c|c|c|c|c|c|c|c|c|c|c|}
\hline & & $\begin{array}{c}\mu_{a i} \\
(m v)\end{array}$ & $\begin{array}{c}\sigma_{a i} \\
(m v)\end{array}$ & $\begin{array}{l}\mu_{i} \\
(s) \\
\end{array}$ & $\begin{array}{l}\sigma_{i} \\
(s)\end{array}$ & $\begin{array}{c}\varphi_{i, 0} \\
(\mathrm{rad})\end{array}$ & $\begin{array}{c}\Delta \varphi_{i} \\
(\mathrm{rad})\end{array}$ & $\begin{array}{c}f_{i} \\
(\mathrm{~Hz})\end{array}$ & $\begin{array}{c}T \\
(s) \\
\end{array}$ & $\begin{array}{l}S N R \\
(d B)\end{array}$ & $K$ \\
\hline \multirow{4}{*}{ 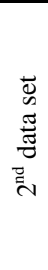 } & $\overline{\mathrm{s}_{1}^{+}}$ & 0.45 & 0.25 & 0.0446 & 0.0223 & 2.83 & $\frac{\pi}{6}$ & 67.86 & \multirow{4}{*}{0.83} & \multirow{4}{*}{30} & \multirow{4}{*}{100} \\
\hline & $\mathrm{s}_{1}^{-}$ & 0.93 & 0.17 & 0.0748 & 0.0111 & 3.14 & $\frac{\pi}{6}$ & 76.59 & & & \\
\hline & $\mathrm{s}_{2}^{+}$ & 0.70 & 0.20 & 0.3676 & 0.0111 & 3.14 & $\frac{\pi}{6}$ & 69.12 & & & \\
\hline & $\mathrm{s}_{2}^{-}$ & 0.51 & 0.09 & 0.3915 & 0.0111 & 0.00 & $\frac{\pi}{6}$ & 62.83 & & & \\
\hline \multirow{4}{*}{ 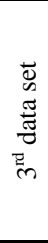 } & $\overline{\mathrm{s}_{1}^{+}}$ & 0.33 & 0.03 & 0.23 & 0.0127 & 0.0127 & $\frac{\pi}{4}$ & 63.21 & \multirow{4}{*}{0.76} & \multirow{4}{*}{20} & \multirow{4}{*}{100} \\
\hline & $\mathrm{s}_{1}^{-}$ & 0.80 & 0.19 & 0.44 & 0.0111 & 0.0111 & $\frac{\pi}{4}$ & 68.05 & & & \\
\hline & $\mathrm{s}_{2}^{+}$ & 0.53 & 0.02 & 2.39 & 0.0127 & 0.0127 & $\frac{\pi}{4}$ & 62.27 & & & \\
\hline & $\mathrm{s}_{2}^{-}$ & 0.52 & 0.05 & 2.36 & 0.0095 & 0.0095 & $\frac{\pi}{4}$ & 66.98 & & & \\
\hline \multirow{4}{*}{ 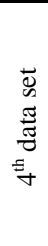 } & $\overline{\mathrm{s}_{1}^{+}}$ & 0.43 & 0.04 & 0.25 & 0.0175 & 2.23 & $\frac{\pi}{3}$ & 65.97 & \multirow{4}{*}{0.65} & \multirow{4}{*}{10} & \multirow{4}{*}{100} \\
\hline & $\mathrm{s}_{1}^{-}$ & 0.76 & 0.15 & 0.50 & 0.0127 & 3.14 & $\frac{\pi}{3}$ & 71.94 & & & \\
\hline & $\mathrm{s}_{2}^{+}$ & 0.43 & 0.03 & 2.36 & 0.0064 & 3.14 & $\frac{\pi}{3}$ & 67.36 & & & \\
\hline & $\mathrm{s}_{2}^{-}$ & 0.33 & 0.08 & 2.45 & 0.0271 & 3.14 & $\frac{\pi}{3}$ & 66.98 & & & \\
\hline
\end{tabular}

$2^{\text {nd }}$ data set

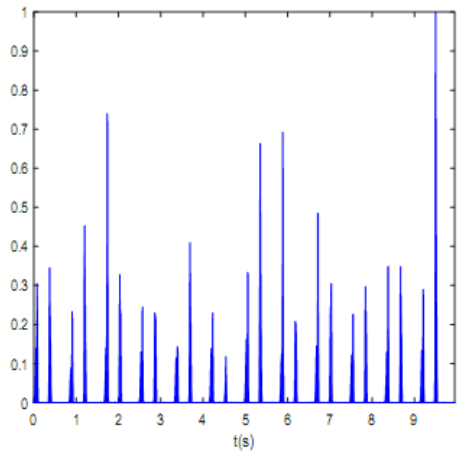

$3^{\text {rd }}$ data set

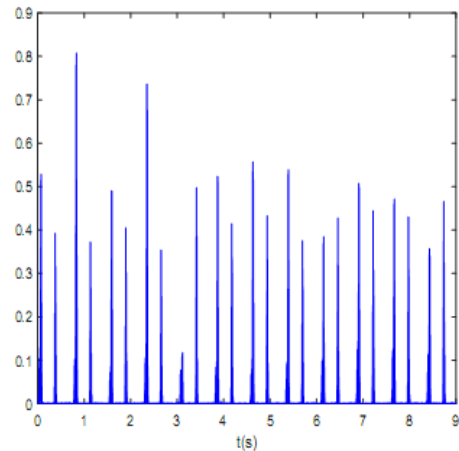

$4^{\text {th }}$ data set

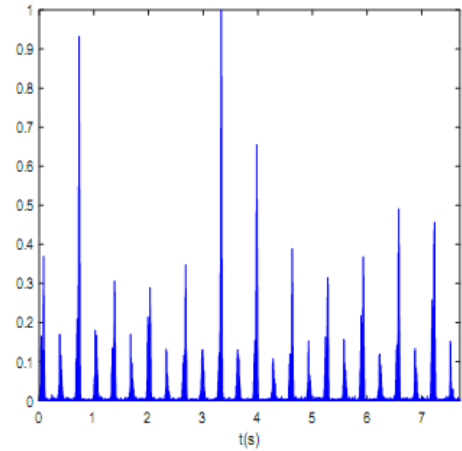

Fig.6. A numerical estimate of the time-varying autocorrelation functions of each synthetic PCG signal.

$2^{\text {nd }}$ data set

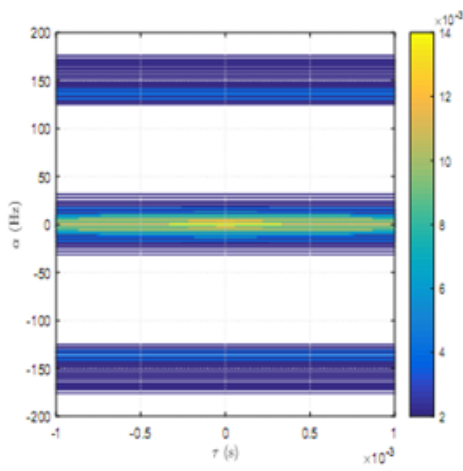

$3^{\text {rd }}$ data set

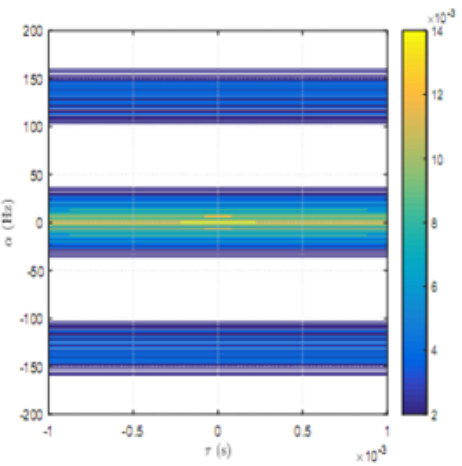

$4^{\text {th }}$ data set

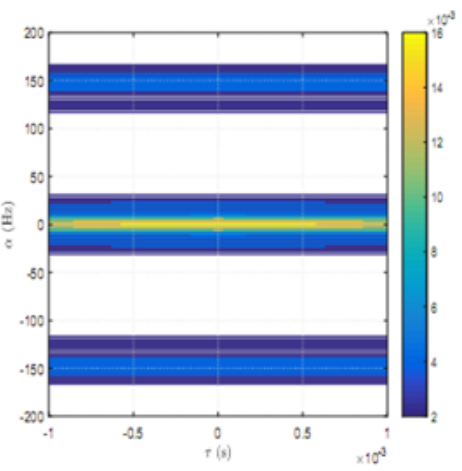



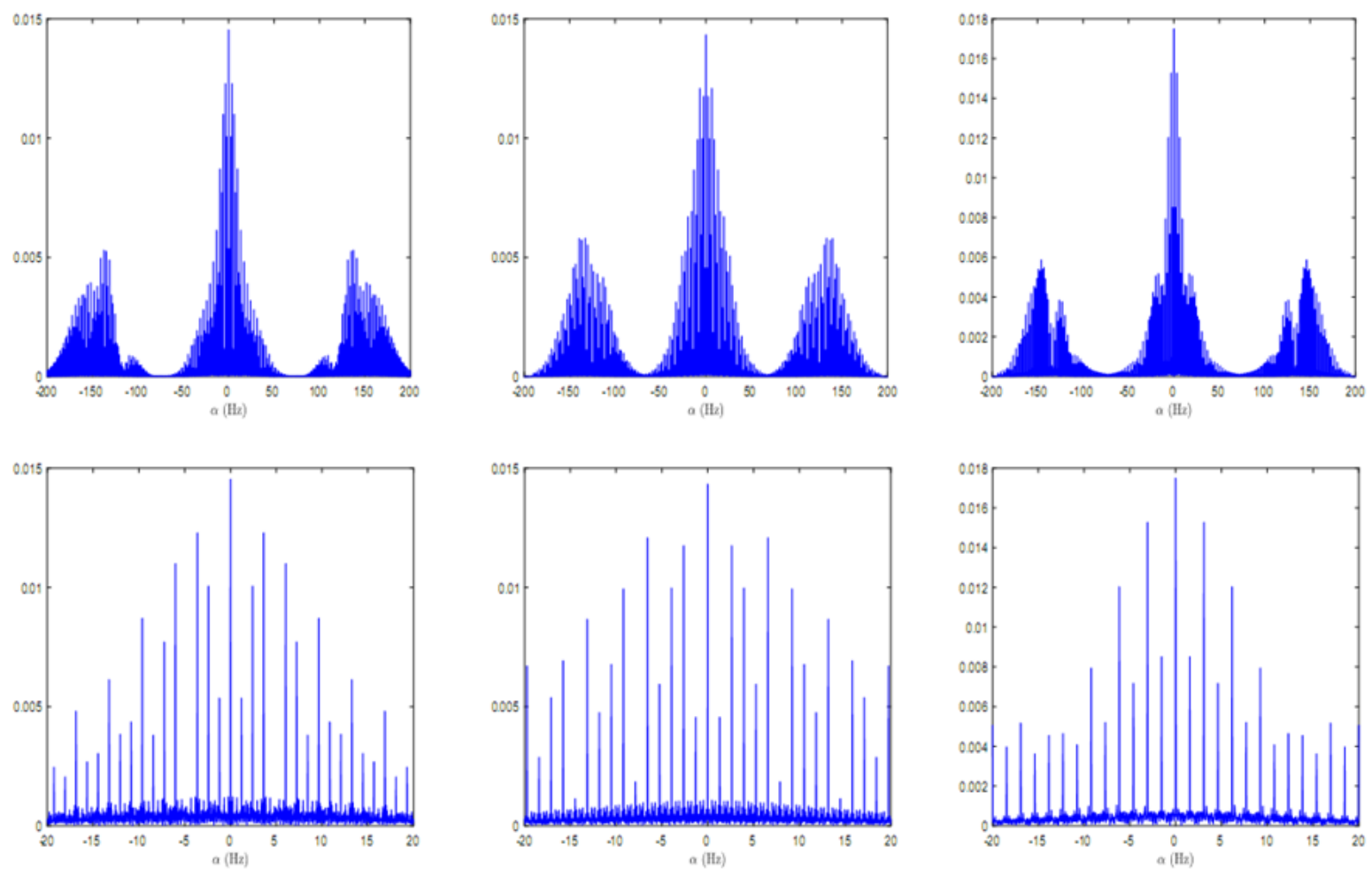

Fig.7. A numerical estimate of the cyclic autocorrelation functions $R_{z}^{\alpha}(\tau): 1^{\text {st }}$ range- $R_{z}^{\alpha}(\tau)$ as a function of $\alpha$ and $\tau$, $2^{\text {nd }}$ range- $R_{z}^{\alpha}(\tau)$ in the $\alpha$ plan for $\tau=0 s$, and $3^{\text {rd }}$ range- zoom of $R_{z}^{\alpha}(\tau)$ in axis $\alpha$ for $\tau=0 s$; of each synthetic PCG signal.

$2^{\text {nd }}$ data set
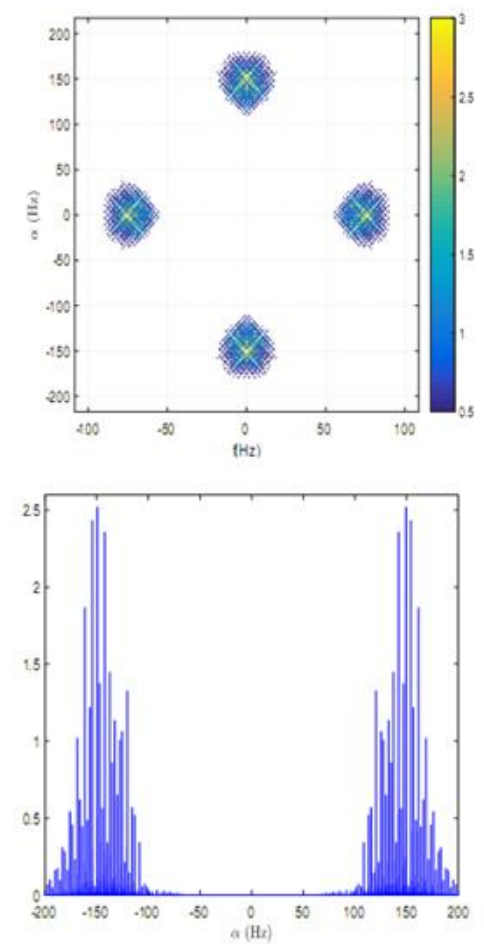

$3^{\text {rd }}$ data set
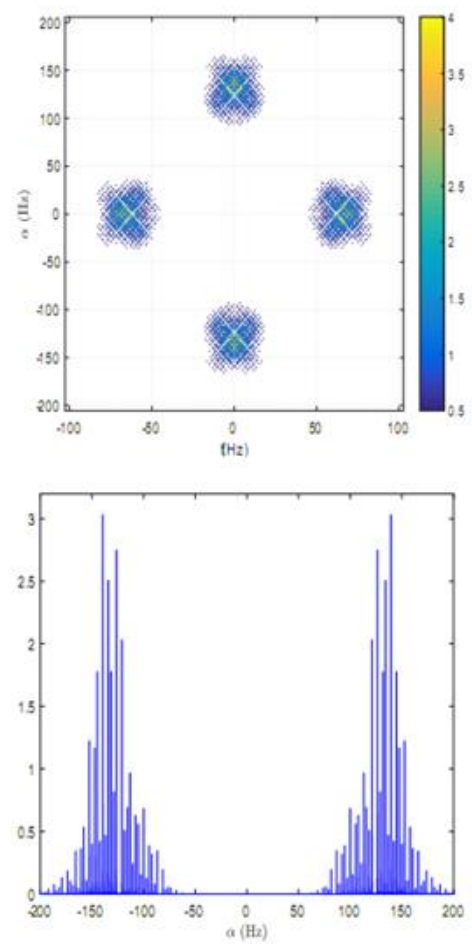

$4^{\text {th }}$ data set
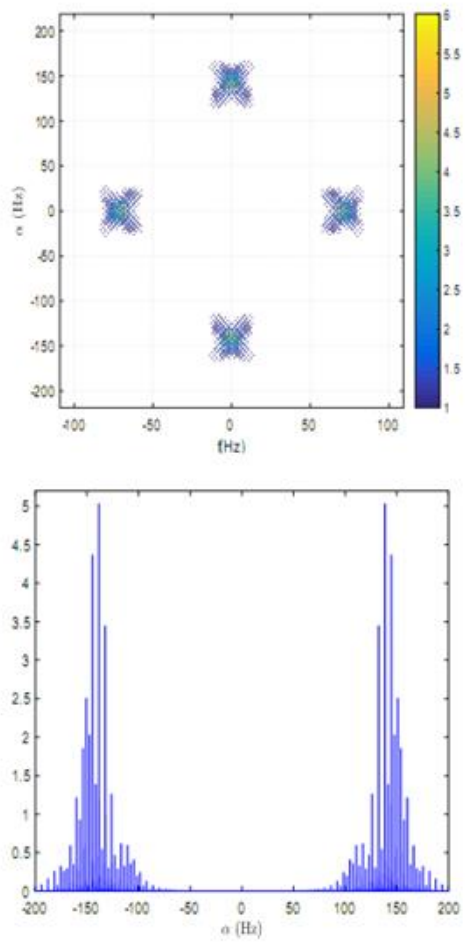

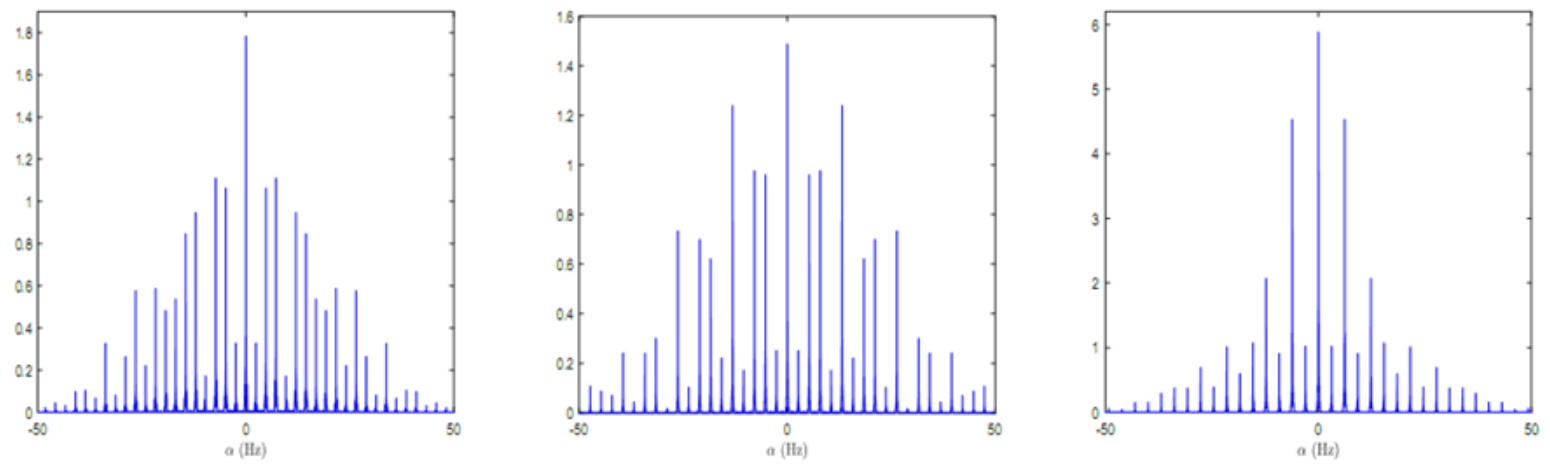

Fig.8. A numerical estimate of the spectral correlation density $S_{z}^{\alpha}(f): 1^{\text {st }}$ range- $S_{z}^{\alpha}(f)$ as a function of $\alpha$ and $\mathrm{f}, 2^{\text {nd }}$ range- $S_{z}^{\alpha}(f)$ in the $\alpha$-plan, for

$f=0 \mathrm{~Hz}$ and $3^{\text {rd }}$ range- zoom in axis $\alpha$ for $(f=76.66 \mathrm{~Hz}, f=67.87 \mathrm{~Hz}, f=71.77 \mathrm{~Hz})$; of each synthetic PCG signal.

The second order statistics reported in Figs. (6, 7 and 8 ), confirm the cyclic behavior of the three PCG signals even if the cyclic periods are different. It should be noted that the cyclic statistics are not sensitive to noise since the noise is supposed to be stationary.

\section{B. Experimental PCG Signals}

The estimations of second order statistics for real PCG signals will indicate the matching of the proposed model of (2) with reality. To do this we make use of data sets provided from [15] which have been gathered from a clinic trial in hospitals using the digital stethoscope DigiScope.
The second order statistics reported in Figs. (9, 10 and 11) show that the three real PCG signals are well widesense cyclostationary. This result matches with the one of synthetic PCG signals which proves the effectiveness of the proposed model of (2). It should be noted that the last two real PCG signals are very noisy. The perturbations in cyclic autocorrelation function and the spectral correlation density of the last two real PCG signals may be explained by the strong fluctuation of the cyclic period i.e. cardiac cycle. Furthermore, the spectral correlation density functions are thresholded in order to exclude noise due to the estimator since signals have a small number of samples.
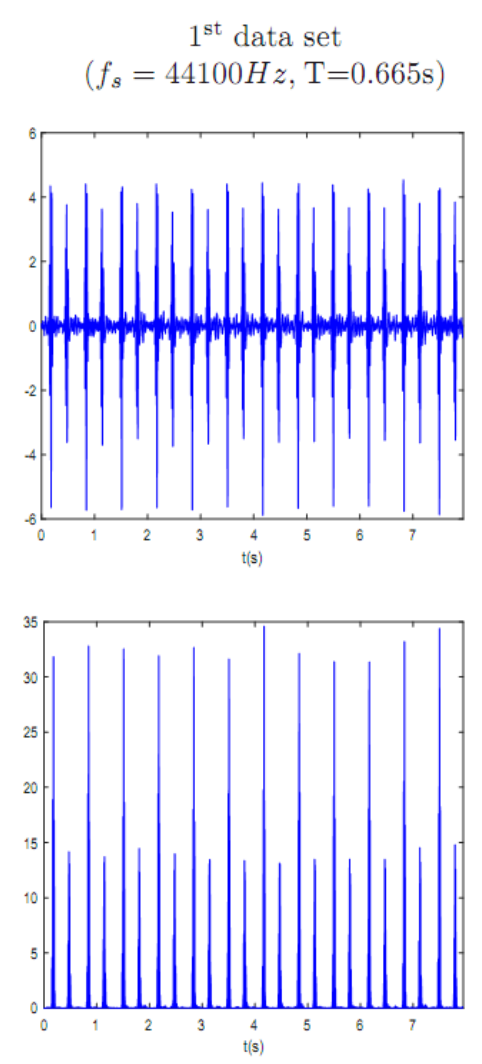
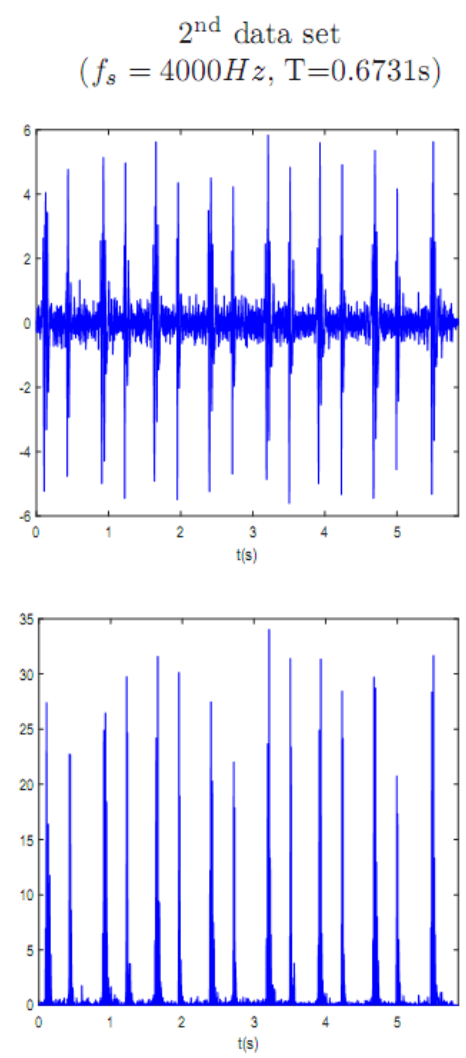

$3^{\text {rd }}$ data set

$\left(f_{s}=4000 H z, \mathrm{~T}=1.04 \mathrm{~s}\right)$
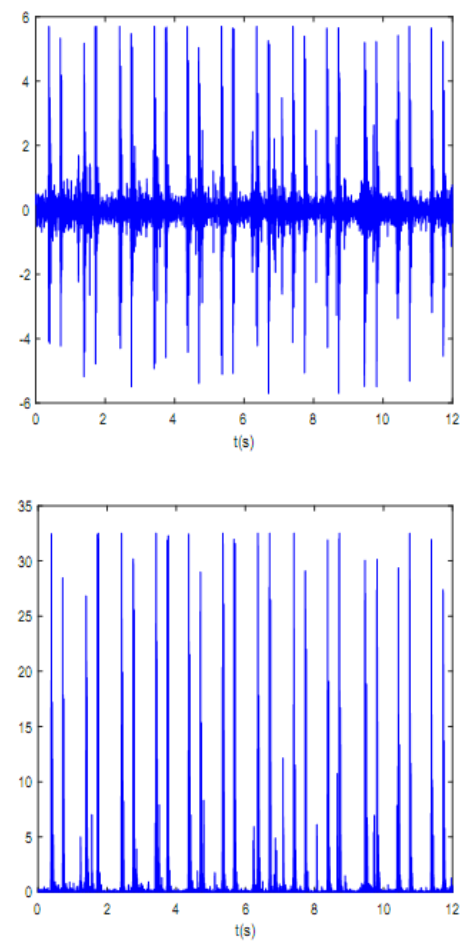

Fig.9. $1^{\text {st }}$ range- time representation of the real PCG signals and $2^{\text {nd }}$ range- time-varying autocorrelation function for each real PCG signal. 
$1^{\text {st }}$ data set
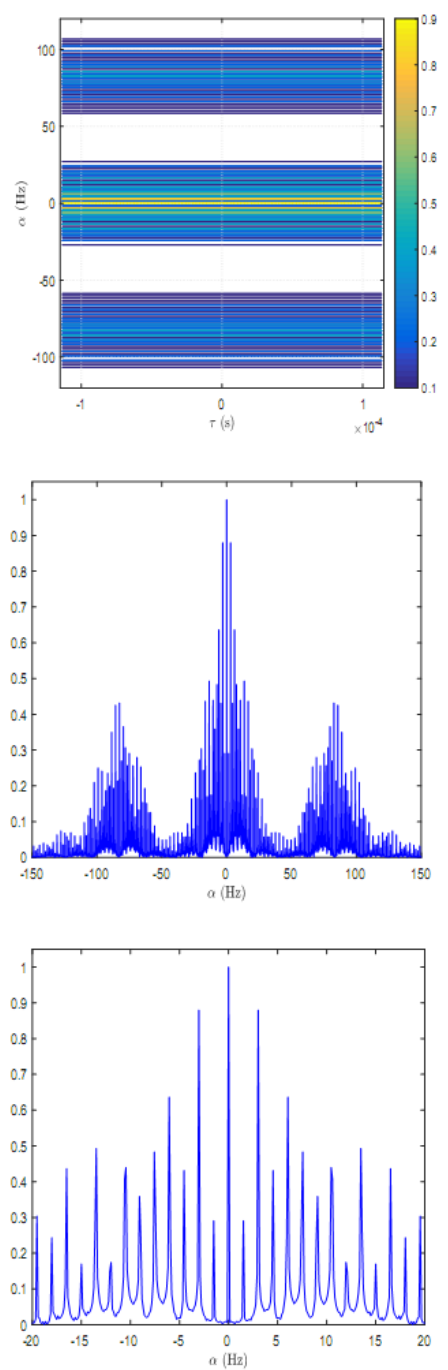

$2^{\text {nd }}$ data set
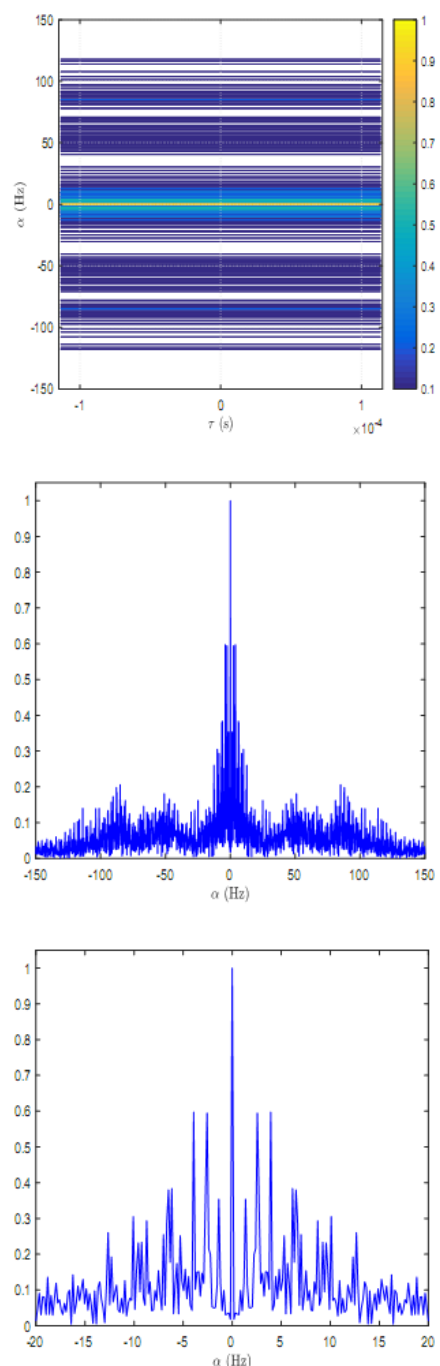

$3^{\text {rd }}$ data set
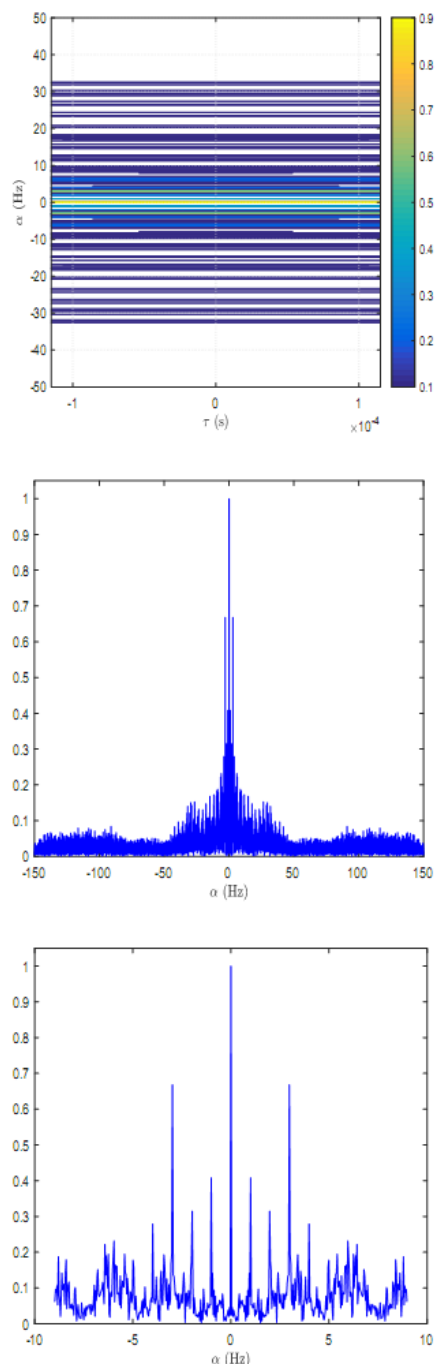

Fig.10. The cyclic autocorrelation functions: $R_{z}^{\alpha}(\tau)$ of the three real PCG signals: $1^{\text {st }}$ range- $R_{z}^{\alpha}(\tau)$ : as a function of $\alpha$ and $\tau, 2^{\text {nd }}$ range- $R_{z}^{\alpha}(\tau)$ in the $\alpha$-plan for $\tau=0 s$ and $3^{\text {rd }}$ range- zoom of $R_{z}^{\alpha}(\tau)$ in axis $\alpha$ for $\tau=0 s$.

$1^{\text {st }}$ data set

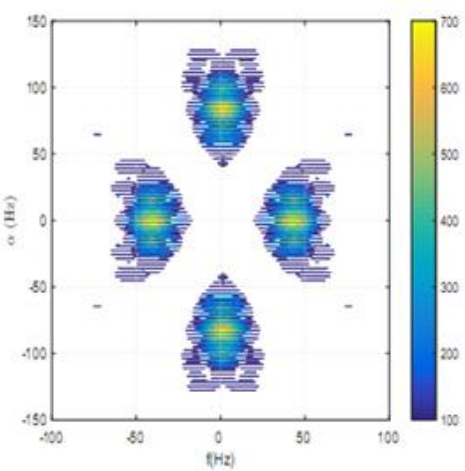

$2^{\text {nd }}$ data set

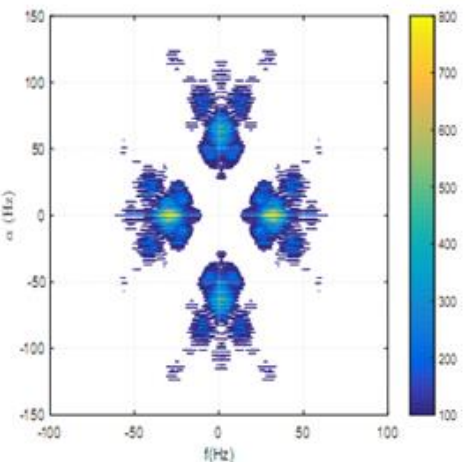

$3^{\text {rd }}$ data set

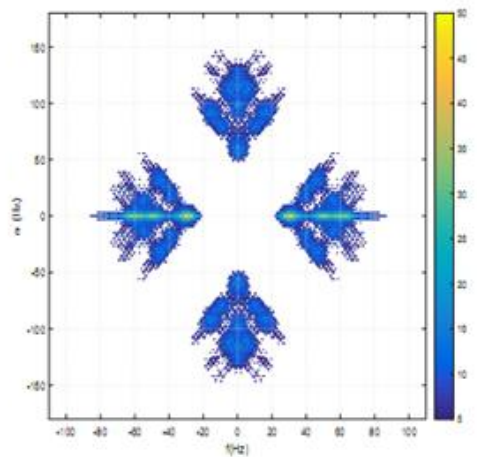



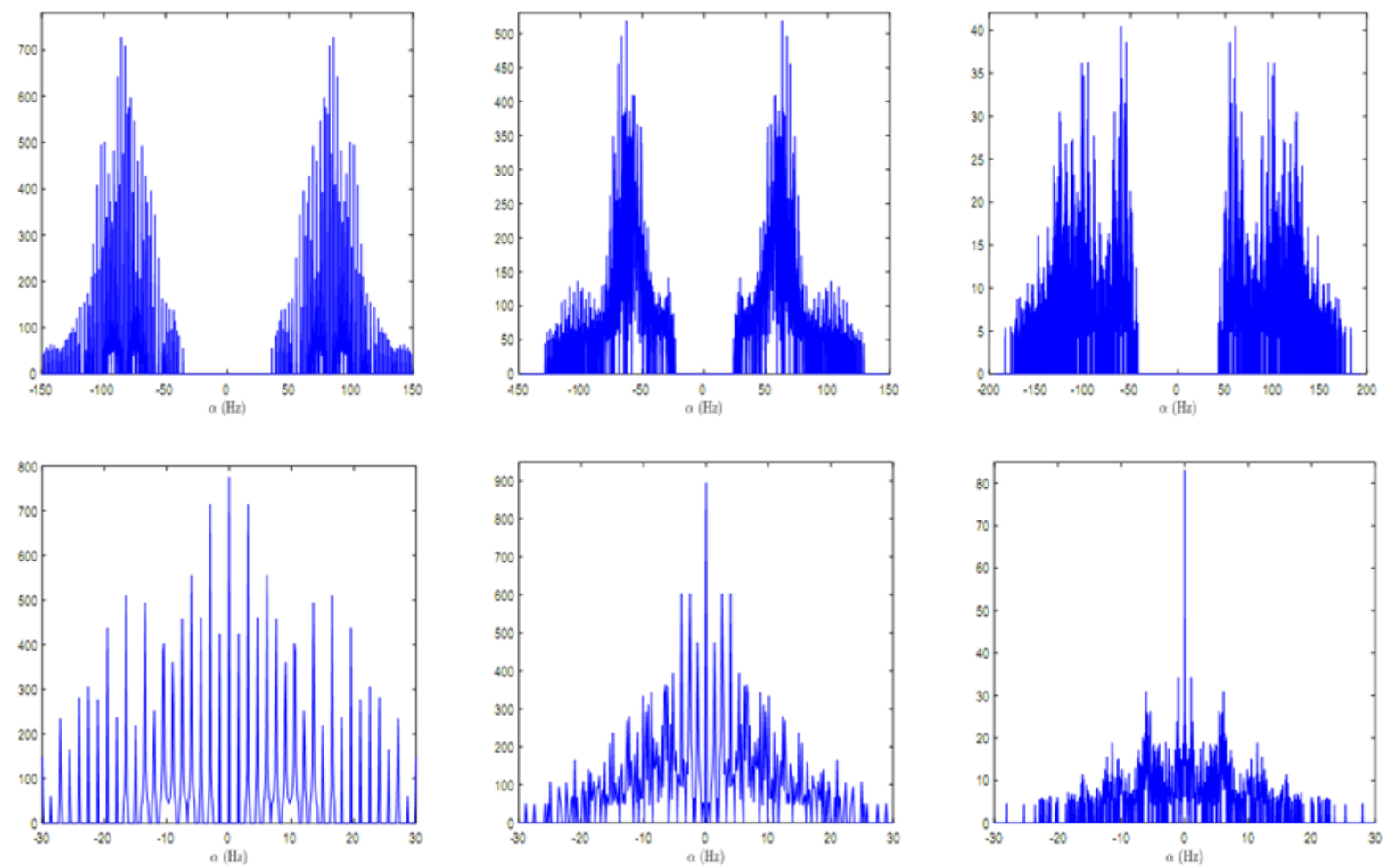

Fig.11. The spectral correlation density $S_{z}^{\alpha}(f)$ of the three real PCG signals: $1^{\text {st }}$ range- $S_{z}^{\alpha}(f)$ as a function of $\alpha$ and f, $2^{\text {nd }}$ range- $S_{z}^{\alpha}(f)$ in the $\alpha$-plan for $f=0 \mathrm{~Hz}$ and $3^{\text {rd }}$ range- zoom in axis $\alpha$ for $(f=43 \mathrm{~Hz}, f=26.91 \mathrm{~Hz}, f=29.29 \mathrm{~Hz})$

\section{CONCLUSION}

In this study, we introduced a new PCG model allowing a better description of heart beats over several cardiac cycles. The effectiveness of the model was validated over synthetic and real PCG signals. We proved the wide-sense cyclostationarity of the model that allows an accurate characterization of PCG signals as cyclic statistics are not influenced by stationary additive noise. Hence, any trouble in the heart functioning, even small, will influence second-order cyclic statistics. This modification could be used as an alarm that alerts cardiologists to suspect early heart abnormalities.

\section{REFERENCES}

[1] W. A. Gardner, Introduction to Random Processes: with applications to signals and systems, Macmillan Publishing Company.New York, 1985.

[2] H. Koymen, B.K. Altay, Y. Z. Ider, A study of prosthetic heart valve sounds, IEEE Transactions on, Biomedical Engineering (11), 853-863, (1987).

[3] W. A. Gardner, Two alternative philosophies for estimation of the parameters of time-series, IEEE Transactions on Information Theory, 37(1), 216-218, (1991).

[4] W. A. Gardner, Cyclostationarity in communications and signal processing, Statistical Signal Processing, Inc Yountville, CA 1994.

[5] A. Baykal, Y. Z. Ider and H. Köymen, Distribution of aortic mechanical prosthetic valve closure sound model parameters on the surface of the chest, IEEE Transactions on, Biomedical Engineering, 42(4), 358-370, (1995).

[6] H. P. Sava, and J. T. E. McDonnell, Modified forwardbackward over determined Prony's method and its application in modelling heart sounds, IEE ProceedingsVision, Image and Signal Processing, 142(6), 375-380, (1995).

[7] H. Sava, P. Pibarot, and L. G. Durand, Application of the matching pursuit method for structural decomposition and averaging of phonocardiographic signals, Medical and Biological Engineering and Computing, 36(3), 302-308, (1998).

[8] X. Zhang, L. G. Durand, L. Senhadji, H. C. Lee, and J. L. Coatrieux, Analysis-synthesis of the phonocardiogram based on the matching pursuit method, IEEE Transactions on Biomedical Engineering, 45(8), 962-971, (1998).

[9] T. S. Leung, P. R. White, J. Cook, W. B. Collis, E. Brown and A. P. Salmon, Analysis of the second heart sound for diagnosis of paediatric heart disease, IEE ProceedingsScience, measurement and technology, 145(6), 285-290, (Nov, 1998).

[10] J. Xu, L. Durand, P. Pibarot, Nonlinear transient chirp signal modeling of the aortic and pulmonary components of the second heart sound, IEEE Transactions on, Biomedical Engineering, 47(10), 1328-1335, (2000).

[11] J. Xu, L. Durand, P. Pibarot, Extraction of the aortic and pulmonary components of the second heart sound using a nonlinear transient chirp signal model, IEEE Transactions on, Biomedical Engineering, 48(3), 277-283, (2001).

[12] S. M. Debbal, and F. Bereksi-Reguig, Time-frequency analysis of the first and the second heartbeat sounds, Applied Mathematics and Computation, 184(2), 10411052, (2007). 
[13] K. Sabri, M. El Badaoui, F. Guillet, A. Belli, G. Millet, and J. B. Morin, Cyclostationary modeling of ground reaction force signals, Signal Processing, vol. 90, no. 4, pp. 1146-1152, April 2010.

[14] A. Almasi, M. B. Shamsollahi, and L. Senhadji, A Dynamical Model for Generating Synthetic Phonocardiogram Signals, In Engineering in Medicine and Biology Society, EMBC, 2011 Annual International Conference of the IEEE (pp. 5686-5689), IEEE, (August, 2011).

[15] P. Bentley, G. Nordehn, M. Coimbra, and S. Mannor, The PASCAL Classifying Heart Sounds Challenge 2011 (CHSC 2011) Results, http//www.peterjbentley.com/heartchallenge/index.html

[16] K. Sabri, Cyclic sparse greedy deconvolution, International Journal of Image, Graphics and Signal Processing, 4(12), 1, 2012.

[17] A. Goshvarpour, A. Goshvarpour, Recurrence plots of heart rate signals during meditation, International Journal of Image, Graphics and Signal Processing, 4(2), 44, (2012).

[18] A. Almasi, M. B. Shamsollahi, and L. Senhadji, Bayesian denoising framework of phonocardiogram based on a new dynamical model, IRBM, 34(3), 214-225, (2013).

[19] M. Jabloun, P. Ravier, O. Buttelli, R. Lédée, R. Harba, and L. D. Nguyen, A generating model of realistic synthetic heart sounds for performance assessment of phonocardiogram processing algorithms, Biomedical Signal Processing and Control, 8(5), 455-465, (2013).

[20] A. Napolitano, Cyclostationary Signal Processing and its Generalizations, IEEE Statistical Signal Processing Workshop, Gold Coast, Australia, June 29, 2014.

\section{Authors' Profiles}

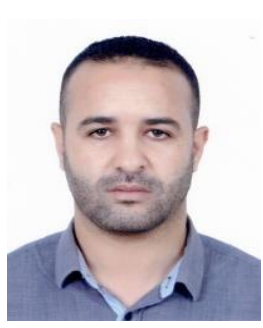

Abdelouahad CHOKLATI received the Licence degree in Electronic, in 2008, and the Master degree in Electronic and Signal Processing, in 2010, from Chouaib Doukkali University of El Jadida, Morocco. He is Professor of Secondary Education (Engineering Sciences, Electrical Engineering). Since January 2012, he is a Ph.D. student in the STIC laboratory, Faculty of sciences, University Chouaib Doukkali, El Jadida, Morocco. His research interest includes Cyclostationarity and Biomedical signal processing.

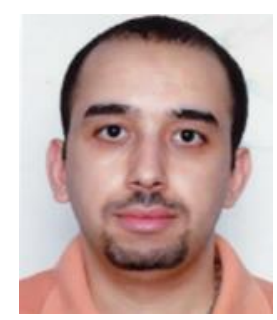

Khalid SABRI was born in Rabat, Morocco on October $28^{\text {th }} 1979$. He received the B. S. degree in informatic, electronic, electrotechnic and automatic in 2002 and the M.S. degree in informatic and telecommunications from the University Mohammed V-Agdal, Rabat, Morocco in 2004, and the Ph.D. degree, in Signal Processing, in September 2008, jointly form the University Jean Monnet, Saint-Etienne, France and the University Mohammed V-Agdal. From April 2006 to September 2006, he was visiting research at the University of New South Wales, Sydney, Australia. From September 2008 to December 2008, he had a post-doc position at Laboratoire d'analyse des signaux et des processus industriels, the University Jean Monnet. From January 2009 to December 2010, he had a post-doc position at Institut de Recherche en Astro physique et Planétologie, Observatoire Midi-Pyrénées, Toulouse, France. Since 2011, he has been an associate professor at the University Chouaiib Doukkali, El Jadida, Morocco. His research interests are in the areas of blind source separation, Sparse Deconvolution, Cyclostationarity, Diagnostic of roatating machines, Biomedical signal proecssing.

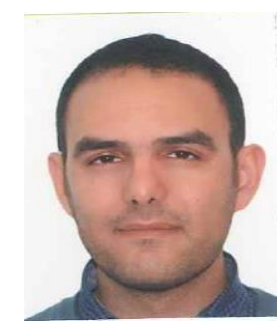

Mounir LAHLIMI holds an engineering degree in electromechanical from National School of Mines of Rabat (Morocco) and a master degree in Optics Image Vision from University Jean Monnet Saint-Etienne (France). A professional engineer, His career of more than 10 years in condition monitoring, is an expert engineering of Dynae, specialized Services Company In the monitoring and diagnosis of machines. His research interests are in the areas of signal processing and diagnostic of rotating machines.

How to cite this paper: A.Choklati, K. Sabri, M. Lahlimi," Cyclic Analysis of Phonocardiogram Signals", International Journal of Image, Graphics and Signal Processing(IJIGSP), Vol.9, No.10, pp.1-11, 2017.DOI: 10.5815/ijigsp.2017.10.01 